\title{
Effect of Azotobacter vinelandii strain SRIAz3 and N-source on Microbiological Properties of Rice Grown Soil
}

\author{
Soumya Ranjan Dehury ${ }^{1}$, Rajeswari Das ${ }^{1 *}$, Pinki Seth ${ }^{2}$, \\ Madhusmita Pradhan ${ }^{1}$ and Santanu Mohanty ${ }^{1}$
}

\author{
${ }^{1}$ Department of Soil Science and Agricultural Chemistry, College of Agriculture OUAT, \\ Bhubaneswar, Odisha, India \\ ${ }^{2}$ Department of Soil Science and Agricultural Chemistry, College of Agriculture, University of \\ Agricultural Sciences, Bangalore - 560065 (Karnataka), India
}

*Corresponding author

\section{A B S T R A C T}

\begin{tabular}{l} 
K e y w o r d s \\
$\begin{array}{l}\text { Azotobacter rice, } \\
\text { Biomass, Urease, } \\
\text { Dehydrogenase, } \\
\text { Phosphatise activity }\end{array}$ \\
Article Info \\
$\begin{array}{l}\text { Accepted: } \\
\text { 20 February } 2018 \\
\text { Available Online: } \\
\text { 10 March } 2018\end{array}$ \\
\hline
\end{tabular}

\section{Introduction}

Rice is the staple food for 60 percent of Indians and source of livelihood for 120-150 million rural households. India is the largest producer of rice being cultivated in 43.95 Mha area with a production of $106.35 \mathrm{Mt}$ and productivity of $2.42 \mathrm{t}$ ha $^{-1}$ (Agricultural Statistics at a Glance, 2014). In Odisha, rice is cultivated in 4.18 Mha area with production
The present investigation was carried out to assess the efficiency as well as effect of bio inoculation of free living $\mathrm{N}_{2}$ fixing bacterial strain (Azotobacter vinelandii strain SRIAz3) with $\mathrm{N}$ sources on enhancement of microbial properties of soil. A. vinelandii strain SRIAz3 was isolated from SRI Field (Central Farm, OUAT, Bhubaneswar) with optimum nitrogenase activity [121.09 $\mathrm{N}_{2}$-ase activity (nmole $\mathrm{C}_{2} \mathrm{H}_{4} \mathrm{mg}^{-1}$ bacteria ${ }^{-1} \mathrm{~h}^{-1}$ )]. Azotobacter inoculated plots significantly influenced the microbial parameters. In Azotobacter innoculated plot with $75 \%$ nitrogen source total heterotrophic bacteria, Azotobacter population, soil microbial biomass carbon, dehydrogenase, urease and phosphatase activity were enhanced by 40, 160, 80, 240, 80, 210 and 250 percent respectively over uninoculated plots. Higher Azotobacter population resulted in increased soil enzymatic (dehydrogenase, urease and phosphatase) activities and microbial biomass carbon. Use of Azotobacter vinelandii strain SRIAz3 isolated from SRI Field as biofertilizer significantly influenced soil microbial biomass and enzymatic activity, which could consequently enhance the rice yield and nutrient (N, P and $\mathrm{K}$ ) uptake. 
challenges to produce the required quantity per unit area because the growing population has ceased the area of the cultivable land. Green revolution of 1960 s contributed a lot to food grain production by use of high inputs particularly chemical fertilizer. The increase in population has led to intensification of agricultural systems. To meet the food requirement, attempts have been made to increase the food production by utilization of synthetic chemicals such as fertilizers and pesticides. This led to many fold increase in food grain production but it has also causedsoil health degradation, environmental pollution and moreover the unsustainable crop production. It is well known that microorganisms play an important role in maintaining soil health in the farming ecosystem. So the activities related to production, preservation and utilization of microorganisms on commercial scale in agriculture sector are fast increasing in recent times.

Biological nitrogen fixation (BNF) has become important in rice farming systems because, this process diminishes the need for expensive chemical fertilizers which have been associated with numerous health and environmental problems. As crop growth and development are closely related to the nature of the soil microflora, especially those in close proximity to plant roots, i.e. the rhizosphere. Therefore control of microorganisms is essentially required to overcome the limitations of conventional agricultural technologies. The soil and rhizosphere microflora can accelerate the growth of plants and enhance their resistance to disease and harmful insects by producing bioactive substances. Biological nitrogen fixation (BNF) has become important in rice farming systems because this process diminishes the need for expensive chemical fertilizers which have been associated with numerous health and environmental problems. Among different microorganisms Azotobacter plays an important role in Biological nitrogen fixation. Azotobacter are aerobic, free-living soil microbes which play an important role in the nitrogen cycle in nature, binding atmospheric nitrogen which is inaccessible to plants and releasing it in the form of ammonium ions into the soil (nitrogen fixation). In addition to being a model organism for studying diazotrophs, it is used by humans for the production of biofertilizers, food additives and some biopolymers.

\section{Materials and Methods}

The techniques of investigation followed and the materials used for the experiments are described in this section.

\section{Field experimentation}

The Field trial was conducted in the Agronomy Research Plot, Orissa University of Agriculture and Technology (OUAT), Bhubaneswar, Odisha located at $20^{\circ} 26^{\prime} \mathrm{N}$ latitude and $85^{\circ} 80^{\prime} \mathrm{E}$ longitude with an altitude of $30 \mathrm{~m}$ above mean sea level, which is $60 \mathrm{~km}$ away from Bay of Bengal. The experimental site experienced high temperature in summer and mild temperature during winter .The annual rainfall was about $1505 \mathrm{~mm}$, out of which more than 85 per cent was received between months of July to October. The field experiment was conducted in Randomized complete block design (RCBD) with eight treatment combinations (Table 1) which were replicated thrice.

Medium duration rice cultivar Pyari was raised in nursery bed with recommended management practices. At the time of final land preparation vermicompost was applied @ $2 \mathrm{t} \mathrm{ha}^{-1}$ to each plot. The test crop received $\mathrm{N}-\mathrm{P}_{2} \mathrm{O}_{5}-\mathrm{K}_{2} \mathrm{O} @ 80-40-40 \mathrm{~kg} \mathrm{ha}^{-1}$ in the form of urea, DAP and MOP respectively. Thirty (30) days old seedlings were uprooted from the nursery bed on the day of transplanting and were dipped in Azotobacter broth 
$\left(10^{10} \mathrm{cfu} \mathrm{ml}^{-1}\right)$ for $2 \mathrm{hrs}$ and kept under shade. The seedlings were then transplanted @ 1-2 seedlings/hill in the main field with the recommended spacing of $20 \mathrm{~cm} \mathrm{X} 10 \mathrm{~cm}$. The crop was harvested when more than $80 \%$ grains turned yellow and were sundried in the field for 3-4 days and threshed. The grains, chaffs and straw yields were recorded after proper sun drying to retain $14 \%$ moisture content in grains.

Representative composite soil samples were collected from all the treatments till harvest. The samples were dried under shade, grinded with wooden hammer and sieved through $2 \mathrm{~mm}$ sieve. The samples were preserved in polythene bags for analysis. Fresh rhizosphere soils were used immediately for estimating microbial parameters before transplanting, 45 DAT, 90 DAT and at harvest.

\section{Method of analysis}

\section{Microbial and chemical analysis of Soil samples}

Soil samples were analyzed for different parameters. Soil reaction was determined in 1: 2.5 soil : water ratio by $\mathrm{pH}$ meter as described by Jackson (1973). Organic carbon content of soil was determined by wet digestion procedure of Walkley and Black as outlined by Page et al., 1982. The soil microbial population (heterotrophic bacteria, Azotobacter) was determined by serial dilution and spread plate technique. Microbial biomass carbon (MBC) was measured as described by Vance et al., (1987).

\section{Soil enzyme activity}

Soil Dehydrogenase assay was measured following the method of reduction of 2, 3,5triphenyltetrazolium chloride (TTC) to the creaming red-colored triphenyl formazan (TPF). The red colour intensity was measured at $485 \mathrm{~nm}$ by Spectrophotometer (Tabatabai,
1982).Soil Urease activity was measured as described by Tabatabai and Bremner, 1972.Acid and alkaline phosphatase activity was measured at $420 \mathrm{~nm}$ by Spectrophotometer (Tabatabai and Bremner, 1969).

\section{Results and Discussion}

The experimental findings of the field experiment and laboratory analysis undertaken during the period of investigation are reported in this chapter.

\section{Microbial and chemical analysis of Soil samples}

\section{Initial chemical and microbial properties of the experimental site}

The initial chemical and biological properties of the experimental site (Table 2) revealed that the soil was strongly acidic $(\mathrm{pH}-4.53$ 4.57 ) in reaction. Organic carbon content of the soil was $0.62-0.68$ per cent. Further the nutrient status of the soil revealed that available $\mathrm{N}, \mathrm{P}$ and $\mathrm{K}$ values ranged 143.0 179.6, $7.1-9.5$ and $139.2-153.3 \mathrm{kgN}, \mathrm{P}_{2} \mathrm{O}_{5}$ and $\mathrm{K}_{2} \mathrm{O}$ ha ${ }^{-1}$ respectively.

Biological properties of the sampled soil revealed that the total heterotrophic bacteria and Azotobacter population of the experimental site were $51-60 \times 10^{4}$ and $21-$ $24 \times 10^{4} \mathrm{CFU} \mathrm{\textrm {g } ^ { - 1 }}$ soil respectively. Soil MBC, soil enzymes viz., dehydrogenase, urease, acid and alkaline phosphatase were $87.60-91.89 \mu \mathrm{g} \mathrm{C} \mathrm{g}^{-1}$ soil, $0.05-0.09 \mu \mathrm{g}$ TPF g ${ }^{-1}$ soil, $35.60-37.96 \mu \mathrm{g} \mathrm{NH}_{4} \mathrm{~N} \mathrm{~g}^{-1}$ soil 2, $0.01-0.10$ and $0.02-0.09 \mu \mathrm{M} \mathrm{PNP} \mathrm{g}^{-1} \mathrm{dw}$ soil respectively.

Soil of the experimental site showed strongly acidic reaction $(\mathrm{pH})$ and medium in organic carbon contents. With regard to plant available nutrients, the experimental site was low in available and medium in available $\mathrm{K}$. 
The soil was low in microbial biomass and enzyme activities (dehydrogenase, urease, acid phosphatase and alkaline phosphatase).

\section{Effect of bioinoculation and $\mathbf{N}$ sources on biological properties of soil}

Soil samples collected from the experimental plots at initial, 45 DAT, 90 DAT and at harvest were analyzed to record microbial population (total heterotrophic bacteria, Azotobacter), MBC and soil enzymatic activities (dehydrogenase, urease, acid phosphatase and alkaline phosphatases).

\section{Total heterotrophic bacteria}

Soil samples collected at initial, 45 DAT, 90 DAT and at harvest were enumerated for bacterial population (Table 3). The treatment $\mathrm{T}_{8}($ A. vinelandii strain SRIAz3 $+100 \% \mathrm{~N})$ recorded highest population of heterotrophic bacteria at 45DAT $\left(172.00 \mathrm{X} 10^{4} \mathrm{CFU} \mathrm{g}^{-1}\right.$ soil) and at harvest $\left(182.00 \times 10^{4} \mathrm{CFU} \mathrm{g}^{-1}\right.$ soil) but at 90 DAT, treatment $\mathrm{T}_{7}(A$. vinelandii strain SRIAz3 $+75 \% \mathrm{~N}$ ) recorded the highest population of heterotrophic bacteria (194.00 X 10 ${ }^{4} \mathrm{CFU} \mathrm{g}^{-1}$ soil). Least population of heterotrophic bacteria was recorded in the control plot throughout the rice growth period $\left(53 \times 10^{4}\right.$ to $129 \times 10^{4}$ CFU g ${ }^{-1}$ soil).

Initial population of total heterotrophic bacteria in all the trial plots were comparatively less than those obtained in successive rice growth period. Further during the active crop growth period i.e. at 45 DAT and 90 DAT the heterotrophic bacteria population in plots inoculated with the free living $\mathrm{N}_{2}$ fixing bacterium (A. vinelandii strain SRIAz3) failed to differ significantly than the uninoculated plots except control. Irrespective of nitrogen doses the inoculated plots maintained total heterotrophic bacteria population at par which may be attributed to the fact that the soil was low in available nitrogen so any addition of nitrogen enhanced immobilization rate which resulted in enhanced total heteroprophic bacterial population which were similar in magnitude. However, in all the treated plots the bacteria population found to decline towards harvest due to mortality of bacteria because of senescence. At harvest total heterotrophic bacteria in the plots inoculated with $A$. vinelandii strain SRIAz3 and receiving nitrogen@75\% and 100\% were found at par and significantly higher over rest of the treatments signifying that the recommended dosage of nitrogen can be reduced under fertilizer scarce conditions.

\section{Azotobacter population}

Soil samples collected at initial, 45 DAT, 90 DAT and at harvest were analyzed for Azotobacter population (Table 4). The treatment $\mathrm{T}_{7}$ (A. vinelandii strain SRIAz3 + $75 \% \mathrm{~N}$ ) with initial population of $23.00 \times 10^{4}$ CFU g ${ }^{-1}$ soil recorded the highest Azotobacter population (111.67, 198.00and $178.33 \times 10^{4}$ CFU $\mathrm{g}^{-1}$ soil) at 45DAT, 90 DAT and at harvest, respectively.However, Azotobacter population was least $(53.00,76.00$ and 67.66 $\mathrm{X} 10^{4} \mathrm{CFU} \mathrm{g}^{-1}$ soil) in the control plot throughout the crop growth period.

Azotobacter population in all the plots at initial stage was comparatively lower than the successive growth stages. At 45 DAT, 90 DAT and at harvest plots inoculated with the free living $\mathrm{N}_{2}$ fixing bacterium (A. vinelandii strain SRIAz3) showed significantly higher Azotobacter population over the uninoculated plots and control. Uninoculated plots receiving varying doses of nitrogen along with control failed to influence the Azotobacter population indicating poor microbial activity due to low initial soil microbial inoculum. Similar findings were obtained by Babajide and Fagbola (2014).

\section{Microbial Biomass Carbon (MBC)}


Soil samples collected periodically at initial, 45 DAT, 90 DAT and at harvest were analysed for MBC (Table 5). Highest MBC (198.23, 278.55 and $292.80 \mu \mathrm{g} \mathrm{C} \mathrm{g}^{-1}$ soil) was recorded in the treatment $\mathrm{T}_{7}$ (A. vinelandii strain SRIAz3 + 75\% N) at 45 DAT, 90 DAT and at harvest respectively over the initial value of $91.42 \mu \mathrm{g} \mathrm{C} \mathrm{g}^{-1}$ soil. MBC was lowest with values of $137.80,154.50$ and $152.00 \mu \mathrm{g}$ $\mathrm{C} \mathrm{g}^{-1}$ soil at all the sampled stages of the crop growth in the control plot.
Soil microbial biomass, which can be reflected by MBC is an important index to assess ability of soil microorganism taking part in the recycling of carbon and their substances in soil (Zeng et al., 2005). Recorded result revealed that initial soil MBC increased with growth of rice till harvest. All throughout the rice growth period the plots receiving only mineral nitrogen maintained significantly poor population with marginal differences among them. Kizilkaya (2009) reported an increase in soil MBC values with increased Azotobacter population.

Table. 1 Treatment details

\begin{tabular}{|l|l|}
\hline $\mathbf{T}_{\mathbf{1}}$ & Control \\
\hline $\mathbf{T}_{\mathbf{2}}$ & $\mathrm{N}_{1}(50 \% \mathrm{~N})$ \\
\hline $\mathbf{T}_{\mathbf{3}}$ & $\mathrm{N}_{2}(75 \% \mathrm{~N})$ \\
\hline $\mathbf{T}_{\mathbf{4}}$ & $\mathrm{N}_{3}(100 \% \mathrm{~N})$ \\
\hline $\mathbf{T}_{\mathbf{5}}$ & A. vinelandii strain SRIAz3 \\
\hline $\mathbf{T}_{\mathbf{6}}$ & A. vinelandii strain SRIAz3 $+\mathrm{N}_{1}$ \\
\hline $\mathbf{T}_{7}$ & A. vinelandii strain SRIAz3 $+\mathrm{N}_{2}$ \\
\hline $\mathbf{T}_{\mathbf{8}}$ & A. vinelandii strain SRIAz3 $+\mathrm{N}_{3}$ \\
\hline
\end{tabular}

Table.2 Initial chemical and microbial properties of soil

\begin{tabular}{|c|c|}
\hline \multicolumn{2}{|l|}{ Soil chemical parameters } \\
\hline pH & $4.53-4.57$ \\
\hline Organic carbon [\%] & $0.62-0.68$ \\
\hline Available $\mathbf{N}\left[\mathrm{kg} \mathrm{ha}^{-1}\right]$ & $143.0-179.6$ \\
\hline Available $P\left[\mathrm{~kg} \mathrm{ha}^{-1}\right]$ & $7.14-9.59$ \\
\hline Available $\mathrm{K}\left[\mathrm{kg} \mathrm{ha}^{-1}\right]$ & $139.2-153.3$ \\
\hline \multicolumn{2}{|l|}{ Soil microbial parameters } \\
\hline Total heterotrophic Bacteria [CFU $\mathrm{g}^{-1}$ soil] & $51-60 \times 10^{4}$ \\
\hline Azotobacter population [CFU g g soil] & $21-24 \times 10^{4}$ \\
\hline Microbial biomass carbon $\left[\mu \mathrm{g} \mathrm{C} \mathrm{g}^{-1}\right.$ soil] & $87.60-91.89$ \\
\hline Dehydrogenase Activity [ $\mu \mathrm{g}$ TPF $\mathrm{g}^{-1}$ soil $^{-1}$ ] & $0.05-0.09$ \\
\hline Urease enzymes $\left(\mu \mathrm{g} \mathrm{NH}_{4} \mathrm{Ng}^{-1} \mathrm{dwt}\right.$ soil $\left.2 \mathrm{~h}^{-1}\right)$ & $35.60-37.96$ \\
\hline Acid Phosphatase enzymes ( $\mu$ M PNP g ${ }^{-1}$ dwt soil $h^{-1}$ ) & $0.01-0.10$ \\
\hline Alkaline Phosphatase enzymes $\left(\mu \mathrm{M}\right.$ PNP $\left.\mathrm{g}^{-1} \mathrm{dwt}_{\text {soil }} \mathrm{h}^{-1}\right)$ & $0.02-0.09$ \\
\hline
\end{tabular}


Table.3 Effect of bioinoculation and $\mathrm{N}$ sources on total heterotrophic bacteria (X 10 ${ }^{4} \mathrm{CFU} \mathrm{g}^{-1}$ soil)

\begin{tabular}{|c|c|c|c|c|}
\hline Treatments & Initial & $45 \mathrm{DAT}$ & $90 \mathrm{DAT}$ & HARVEST \\
\hline Control & $53.00^{\mathrm{a}}$ & $124.00^{\mathrm{b}}$ & $138.00^{\mathrm{b}}$ & $129.00^{c}$ \\
\hline $\mathrm{N} 1(50 \% \mathrm{~N})$ & $51.00^{\mathrm{a}}$ & $147.00^{\mathrm{ab}}$ & $165.00^{\mathrm{ab}}$ & $153.00^{b}$ \\
\hline $\mathrm{N} 2(75 \% \mathrm{~N})$ & $57.00^{\mathrm{a}}$ & $144.00^{\mathrm{ab}}$ & $167.00^{\mathrm{ab}}$ & $158.00^{\mathrm{b}}$ \\
\hline N3 $(100 \% \mathrm{~N})$ & $54.00^{\mathrm{a}}$ & $156.00^{\mathrm{a}}$ & $170.00^{\mathrm{a}}$ & $154.00^{b}$ \\
\hline A. vinelandii strain SRIAz3 & $52.00^{\mathrm{a}}$ & $154.00^{\mathrm{a}}$ & $176.00^{\mathrm{a}}$ & $159.00^{b}$ \\
\hline A. vinelandii strain SRIAz3 + N1 & $54.00^{\mathrm{a}}$ & $169.00^{\mathrm{a}}$ & $182.00^{\mathrm{a}}$ & $165.00^{\mathrm{b}}$ \\
\hline A. vinelandii strain SRIAz3 + N2 & $60.00^{\mathrm{a}}$ & $168.00^{\mathrm{a}}$ & $194.00^{\mathrm{a}}$ & $178.00^{\mathrm{a}}$ \\
\hline A. vinelandii strain SRIAz3 + N3 & $58.00^{\mathrm{a}}$ & $172.00^{\mathrm{a}}$ & $192.00^{\mathrm{a}}$ & $182.00^{\mathrm{a}}$ \\
\hline CV (\%) & 10.59 & 10.14 & 9.43 & 4.03 \\
\hline
\end{tabular}

*Means averaged over three replicates represented by the same letter in columns are not significantly different $(\mathrm{p}<0.05)$.

Table.4 Effect of bioinoculation and $\mathrm{N}$ sources on population of Azotobacter (X $10^{4} \mathrm{CFU} \mathrm{g}^{-1}$ soil)

\begin{tabular}{|l|c|c|c|c|}
\hline Treatments & Initial & 45 DAT & 90 DAT & HARVEST \\
\hline Control & $21.00^{\mathrm{a}}$ & $53.00^{\mathrm{b}}$ & $76.00^{\mathrm{b}}$ & $67.66^{\mathrm{b}}$ \\
\hline N1 (50\% N) & $22.00^{\mathrm{a}}$ & $60.67^{\mathrm{b}}$ & $91.00^{\mathrm{b}}$ & $65.00^{\mathrm{b}}$ \\
\hline $\mathbf{N 2}(\mathbf{7 5 \%} \mathbf{N})$ & $23.00^{\mathrm{a}}$ & $68.34^{\mathrm{b}}$ & $101.34^{\mathrm{b}}$ & $73.00^{\mathrm{b}}$ \\
\hline $\mathbf{N 3}(\mathbf{1 0 0 \%} \mathbf{N})$ & $22.00^{\mathrm{a}}$ & $67.00^{\mathrm{b}}$ & $97.67^{\mathrm{b}}$ & $57.33^{\mathrm{b}}$ \\
\hline A. vinelandii strain SRIAz3 & $24.00^{\mathrm{a}}$ & $97.00^{\mathrm{a}}$ & $173.00^{\mathrm{a}}$ & $154.33^{\mathrm{a}}$ \\
\hline A. vinelandii strain SRIAz3 + N1 & $22.00^{\mathrm{a}}$ & $104.00^{\mathrm{a}}$ & $176.00^{\mathrm{a}}$ & $163.33^{\mathrm{a}}$ \\
\hline A. vinelandii strain SRIAz3 + N2 & $23.00^{\mathrm{a}}$ & $111.67^{\mathrm{a}}$ & $198.00^{\mathrm{a}}$ & $178.33^{\mathrm{a}}$ \\
\hline A. vinelandii strain SRIAz3 + N3 & $22.00^{\mathrm{a}}$ & $98.67^{\mathrm{a}}$ & $165.00^{\mathrm{a}}$ & $134.00^{\mathrm{a}}$ \\
\hline CV $(\%)$ & 8.05 & 10.89 & 15.82 & 26.39 \\
\hline
\end{tabular}

*Means averaged over three replicates represented by the same letter in columns are not significantly different $(\mathrm{p}<0.05)$.

Table.5 Effect of bioinoculation and N sources on Microbial Biomass Carbon $\left(\mu \mathrm{g} \mathrm{C} \mathrm{g}^{-1}\right.$ soil)

\begin{tabular}{|c|c|c|c|c|}
\hline Treatments & Initial & 45 DAT & 90 DAT & HARVEST \\
\hline Control & $87.60^{\mathrm{a}}$ & $137.80^{\mathrm{c}}$ & $154.50^{d}$ & $152.00^{\mathrm{d}}$ \\
\hline $\mathrm{N} 1(50 \% \mathrm{~N})$ & $90.00^{\mathrm{a}}$ & $165.96^{\mathrm{b}}$ & $173.44^{\text {cd }}$ & $187.00^{\text {cd }}$ \\
\hline $\mathrm{N} 2(75 \% \mathrm{~N})$ & $90.00^{\mathrm{a}}$ & $170.54^{\mathrm{b}}$ & $185.32^{\mathrm{cd}}$ & $192.50^{\mathrm{c}}$ \\
\hline N3 $(100 \% \mathrm{~N})$ & $89.34^{\mathrm{a}}$ & $170.45^{\mathrm{b}}$ & $173.68^{\mathrm{cd}}$ & $183.23^{\text {cd }}$ \\
\hline A. vinelandii strain SRIAz3 & $89.84^{\mathrm{a}}$ & $185.77^{\mathrm{ab}}$ & $245.50^{\mathrm{ab}}$ & $254.94^{\mathrm{b}}$ \\
\hline A. vinelandii strain SRIAz3 + N1 & $90.37^{\mathrm{a}}$ & $197.60^{\mathrm{a}}$ & $268.69^{\mathrm{ab}}$ & $283.74^{\mathrm{ab}}$ \\
\hline A. vinelandii strain SRIAz3 + N2 & $91.42^{\mathrm{a}}$ & $198.23^{\mathrm{a}}$ & $278.55^{\mathrm{a}}$ & $292.80^{\mathrm{a}}$ \\
\hline A. vinelandii strain SRIAz3 + N3 & $91.89^{\mathrm{a}}$ & $190.50^{\mathrm{ab}}$ & $221.36^{\mathrm{bc}}$ & $274.94^{\mathrm{ab}}$ \\
\hline CV $(\%)$ & 2.71 & 7.93 & 12.94 & 9.00 \\
\hline
\end{tabular}

*Means averaged over three replicates represented by the same letter in columns are not significantly different $(\mathrm{p}<0.05)$. 
Table.6 Effect of bioinoculation and N sources on Dehydrogenase $\left(\mu \mathrm{g} \mathrm{TPF} \mathrm{g}^{-1}\right.$ soil h$\left.^{-1}\right)$

\begin{tabular}{|l|c|c|c|c|}
\hline Treatments & Initial & 45 DAT & 90 DAT & HARVEST \\
\hline Control & $0.05^{\mathrm{a}}$ & $0.14^{\mathrm{d}}$ & $0.25^{\mathrm{d}}$ & $0.15^{\mathrm{c}}$ \\
\hline $\mathbf{N 1}(\mathbf{5 0 \%} \mathbf{N})$ & $0.05^{\mathrm{a}}$ & $0.23^{\mathrm{cd}}$ & $0.34^{\mathrm{cd}}$ & $0.21^{\mathrm{de}}$ \\
\hline $\mathbf{N} 2(\mathbf{7 5 \%} \mathbf{N})$ & $0.05^{\mathrm{a}}$ & $0.29^{\mathrm{c}}$ & $0.39^{\mathrm{c}}$ & $0.32^{\mathrm{cd}}$ \\
\hline $\mathbf{N 3}(\mathbf{1 0 0 \%} \mathbf{N})$ & $0.07^{\mathrm{a}}$ & $0.29^{\mathrm{c}}$ & $0.42^{\mathrm{c}}$ & $0.40^{\mathrm{c}}$ \\
\hline A. vinelandii strain SRIAz3 & $0.06^{\mathrm{a}}$ & $0.38^{\mathrm{b}}$ & $0.65^{\mathrm{b}}$ & $0.58^{\mathrm{b}}$ \\
\hline $\boldsymbol{A}$. vinelandii strain SRIAz3 + N1 & $0.07^{\mathrm{a}}$ & $0.40^{\mathrm{b}}$ & $0.70^{\mathrm{b}}$ & $0.64^{\mathrm{b}}$ \\
\hline A. vinelandii strain SRIAz3 + N2 & $0.07^{\mathrm{a}}$ & $0.62^{\mathrm{a}}$ & $0.85^{\mathrm{a}}$ & $0.80^{\mathrm{a}}$ \\
\hline $\boldsymbol{A}$. vinelandii strain SRIAz3 + N3 & $0.05^{\mathrm{a}}$ & $0.60^{\mathrm{a}}$ & $0.68^{\mathrm{b}}$ & $0.62^{\mathrm{b}}$ \\
\hline CV $(\%)$ & 21.84 & 13.82 & 14.63 & 17.58 \\
\hline
\end{tabular}

*Means averaged over three replicates represented by the same letter in columns are not significantly different $(\mathrm{p}<0.05)$.

Table.7 Effect of bioinoculation and $\mathrm{N}$ sources on Urease enzyme $\left(\mu \mathrm{g} \mathrm{NH}{ }_{4} \mathrm{~N} \mathrm{~g}^{-1}\right.$ dwt soil $\left.2 \mathrm{~h}^{-1}\right)$

\begin{tabular}{|c|c|c|c|c|}
\hline Treatments & Initial & $45 \mathrm{DAT}$ & $90 \mathrm{DAT}$ & HARVEST \\
\hline Control & $35.60^{\mathrm{a}}$ & $42.30^{\mathrm{d}}$ & $40.56^{\mathrm{e}}$ & $38.90^{\mathrm{e}}$ \\
\hline N1 $(50 \%$ N) & $35.99^{\mathrm{a}}$ & $50.63^{\mathrm{d}}$ & $48.72^{\mathrm{de}}$ & $43.63^{\mathrm{de}}$ \\
\hline N2 $(75 \% N)$ & $36.24^{\mathrm{a}}$ & $62.39^{c}$ & $54.81^{\mathrm{cd}}$ & $49.36^{\mathrm{cd}}$ \\
\hline N3 $(100 \% \mathrm{~N})$ & $36.95^{\mathrm{a}}$ & $63.50^{c}$ & $54.81^{\mathrm{cd}}$ & $52.50^{\text {bcd }}$ \\
\hline A. vinelandii strain SRIAz3 & $37.07^{\mathrm{a}}$ & $67.50^{c}$ & $60.90^{\mathrm{bc}}$ & $59.60^{\mathrm{ab}}$ \\
\hline A. vinelandii strain SRIAz3 + N1 & $37.24^{\mathrm{a}}$ & $68.50^{c}$ & $60.90^{\mathrm{bc}}$ & $53.50^{\mathrm{bc}}$ \\
\hline A. vinelandii strain SRIAz3 + N2 & $37.48^{\mathrm{a}}$ & $89.56^{\mathrm{a}}$ & $73.08^{\mathrm{a}}$ & $66.35^{\mathrm{a}}$ \\
\hline A. vinelandii strain SRIAz3 + N3 & $37.96^{\mathrm{a}}$ & $78.60^{\mathrm{b}}$ & $66.99^{\mathrm{ab}}$ & $54.68^{\mathrm{bc}}$ \\
\hline CV (\%) & 5.03 & 8.88 & 8.42 & 10.13 \\
\hline
\end{tabular}

*Means averaged over three replicates represented by the same letter in columns are not significantly different $(\mathrm{p}<0.05)$.

Table.8 Effect of bioinoculation and $\mathrm{N}$ sources on acid phosphatase enzymes $\left(\mu \mathrm{M}\right.$ PNP g ${ }^{-1}$ dwt soil $\left.\mathrm{h}^{-1}\right)$

\begin{tabular}{|l|c|c|c|c|}
\hline Treatments & Initial & 45 DAT & 90 DAT & HARVEST \\
\hline Control & $0.06^{\mathrm{a}}$ & $0.08^{\mathrm{d}}$ & $0.10^{\mathrm{c}}$ & $0.05^{\mathrm{c}}$ \\
\hline $\mathbf{N} 1(\mathbf{5 0 \%} \mathbf{N})$ & $0.06^{\mathrm{a}}$ & $0.10^{\mathrm{d}}$ & $0.13^{\mathrm{bc}}$ & $0.05^{\mathrm{c}}$ \\
\hline $\mathbf{N} 2 \mathbf{7 5 \%} \mathbf{N})$ & $0.06^{\mathrm{a}}$ & $0.10^{\mathrm{d}}$ & $0.11^{\mathrm{c}}$ & $0.07^{\mathrm{bc}}$ \\
\hline $\mathbf{N 3}(\mathbf{1 0 0 \%} \mathbf{N})$ & $0.06^{\mathrm{a}}$ & $0.15^{\mathrm{c}}$ & $0.19^{\mathrm{b}}$ & $0.13^{\mathrm{b}}$ \\
\hline A. vinelandii strain SRIAz3 & $0.06^{\mathrm{a}}$ & $0.20^{\mathrm{b}}$ & $0.29^{\mathrm{a}}$ & $0.22^{\mathrm{a}}$ \\
\hline A. vinelandii strain SRIAz3 + N1 & $0.06^{\mathrm{a}}$ & $0.23^{\mathrm{b}}$ & $0.30^{\mathrm{a}}$ & $0.26^{\mathrm{a}}$ \\
\hline A. vinelandii strain SRIAz3 + N2 & $0.06^{\mathrm{a}}$ & $0.30^{\mathrm{a}}$ & $0.31^{\mathrm{a}}$ & $0.27^{\mathrm{a}}$ \\
\hline A. vinelandii strain SRIAz3 + N3 & $0.06^{\mathrm{a}}$ & $0.25^{\mathrm{b}}$ & $0.30^{\mathrm{a}}$ & $0.24^{\mathrm{a}}$ \\
\hline CV $(\%)$ & 13.60 & 16.34 & 17.66 & 22.66 \\
\hline
\end{tabular}

*Means averaged over three replicates represented by the same letter in columns are not significantly different $(\mathrm{p}<0.05)$. 
Table.9 Effect of bioinoculation and $\mathrm{N}$ sources on Alkaline phosphatase enzymes $\left(\mu \mathrm{M}\right.$ PNP g ${ }^{-1}$ dwt soil h $\left.{ }^{-1}\right)$

\begin{tabular}{|c|c|c|c|c|}
\hline Treatments & Initial & $45 \mathrm{DAT}$ & $90 \mathrm{DAT}$ & HARVEST \\
\hline Control & $0.07^{\mathrm{a}}$ & $0.07^{\mathrm{e}}$ & $0.08^{\mathrm{f}}$ & $0.05^{\mathrm{e}}$ \\
\hline $\mathrm{N} 1(50 \% \mathrm{~N})$ & $0.06^{\mathrm{a}}$ & $0.08^{\mathrm{de}}$ & $0.12^{\mathrm{e}}$ & $0.06^{\mathrm{de}}$ \\
\hline $\mathrm{N} 2(75 \% \mathrm{~N})$ & $0.06^{\mathrm{a}}$ & $0.12^{\text {cd }}$ & $0.14^{\mathrm{e}}$ & $0.09^{d}$ \\
\hline $\mathrm{N} 3(100 \% \mathrm{~N})$ & $0.07^{\mathrm{a}}$ & $0.14^{\mathrm{bc}}$ & $0.17^{d}$ & $0.13^{c}$ \\
\hline A. vinelandii strain SRIAz3 & $0.07^{\mathrm{a}}$ & $0.15^{\mathrm{bc}}$ & $0.19^{d}$ & $0.16^{\mathrm{bc}}$ \\
\hline A. vinelandii strain SRIAz3 + N1 & $0.08^{\mathrm{a}}$ & $0.17^{b}$ & $0.24^{c}$ & $0.19^{b}$ \\
\hline A. vinelandii strain SRIAz3 + N2 & $0.08^{\mathrm{a}}$ & $0.25^{\mathrm{a}}$ & $0.28^{b}$ & $0.26^{\mathrm{a}}$ \\
\hline A. vinelandii strain SRIAz3 + N3 & $0.07^{\mathrm{a}}$ & $0.24^{\mathrm{a}}$ & $0.33^{\mathrm{a}}$ & $0.23^{\mathrm{a}}$ \\
\hline CV $(\%)$ & 19.60 & 16.55 & 8.90 & 14.70 \\
\hline
\end{tabular}

*Means averaged over three replicates represented by the same letter in columns are not significantly different $(\mathrm{p}<0.05)$

\section{Soil enzyme activity}

Soils sampled periodically at initial, 45 DAT, 90 DAT and at harvest were analysed for Dehydrogenase activity (Table 6). Dehydrogenase activity was highest in the treatment $\mathrm{T}_{7}($ A. vinelandii strain SRIAz3 $+\mathrm{N} 2)$ throughout the crop growth period i.e. 0.62, 0.85 and $0.80 \mu \mathrm{g} \mathrm{TPF} \mathrm{g}^{-1}$ soil $\mathrm{hr}^{-1}$ at 45DAT, 90 DAT and at harvest, respectiviely. Least dehydrogenase activity $(0.14,0.25$ and $0.15 \mu \mathrm{g}$ TPF $\mathrm{g}^{-1}$ soil $\left.\mathrm{h}^{-1}\right)$ was recorded in the control $\left(\mathrm{T}_{1}\right)$ plot during the crop growth period. The initial Dehydrogenase activity in all the plots ranged between $0.05-0.07 \mu \mathrm{g}$ TPF g ${ }^{-1}$ soil $\mathrm{h}^{-1}$.

Soil samples collected at periodic interval (45 DAT and 90 DAT and at harvest) were analyzed for Urease enzyme (Table 7). Significantly higher urease activity (89.56, 73.08 and $66.35 \mu \mathrm{g} \mathrm{NH}_{4} \mathrm{~N} \mathrm{~g}^{-1} \mathrm{dwt}$ soil $\left.2 \mathrm{hr}^{-1}\right)$ was recorded in treatment $\mathrm{T}_{7}(A$. vinelandii strain SRIAz3 $+75 \% \mathrm{~N}$ ) at 45DAT, 90 DAT and at harvest, respectively. Control plot maintained lowest Urease activity of (42.30, 40.56 and $38.90 \mu \mathrm{g} \mathrm{NH}_{4} \mathrm{~N} \mathrm{~g}^{-1} \mathrm{dw}$ soil $2 \mathrm{hr}^{-1}$ ) during the crop growth period. The initial urease activity in all the plots ranged between $35.60-37.96 \mu \mathrm{g} \mathrm{NH}{ }_{4} \mathrm{Ng}^{-1} \mathrm{dw}$ soil $2 \mathrm{~h}^{-1}$.

Soils sampled periodically at initial, 45 DAT, 90 DAT and at harvest were analyzed for Acid phosphatase activity (Table 8). Acid phosphatase activity $(0.30,0.31$ and $0.27 \mu \mathrm{M}$ PNP $\mathrm{g}^{-1}$ dwt soil $\mathrm{h}^{-1}$ ) was highest in the treatment $\mathrm{T}_{7}$ (A. vinelandii strain SRIAz3 + $75 \% \mathrm{~N})$ at 45DAT, 90 DAT and at harvest respectively. Lowest acid phosphatase activity (0.08, 0.10 and $0.05 \mu \mathrm{M}$ PNP g $\mathrm{g}^{-1} \mathrm{dwt}$ soil $\mathrm{h}^{-1}$ ) was obtained in the control (T1) plot during the crop growth period.

Alkaline phosphatase activity $(0.25,0.26 \mu \mathrm{M}$ PNP $\mathrm{g}^{-1} \mathrm{dwt}$ soil $\mathrm{h}^{-1}$ ) was highest in the treatment $\mathrm{T}_{7}(A$. vinelandii strain SRIAz3 + $75 \% \mathrm{~N})$ at 45 DAT and harvest respectively where as at 90 DAT it was highest $(0.33 \mu \mathrm{M}$ PNP $\mathrm{g}^{-1} \mathrm{dwt}$ soil $\mathrm{h}^{-1}$ ) in $\mathrm{T}_{8}$ (A. vinelandii strain SRIAz3 $+100 \% \mathrm{~N}$ ). Lowest alkaline phosphatase activity $(0.07,0.08$ and $0.05 \mu \mathrm{M}$ PNP $\mathrm{g}^{-1} \mathrm{dwt}$ soil $\mathrm{h}^{-1}$ ) was obtained in the control plot during the crop growth period. The initial alkaline phosphatase activity in all the plots ranged between $0.06-0.08 \mu \mathrm{M}$ PNP g ${ }^{-1} \mathrm{dwt}$ soil $\mathrm{h}^{-1}$ (Table 9).

Soil enzymes are soil active protein, and on the other hand soil microbial exudation. At 45DAT,90DAT and at harvest significant difference was observed between the bioinoculated plots and uninoculated plots with respect to dehydrogenase, urease, acid and alkaline phosphatase. Furthermore, the above enzymatic activities showed a declining trend 
towards harvest owing to less root activity. When rice life activity was at strong stages, its roots exudation and soil enzymatic activities were increased up to certain extent. When rice was at the maturity stage, its roots decrepitude and its exudation reduced. As a result, soil enzymatic activities and the restriction to soil microbe activities were weakened. Therefore, soil enzymatic activities as well as soil microbial biomass were reduced. Dehydrogenase, produced by live organism, promoting soil organic matter mineralization, is also an important index to evaluate soil organic matter decomposing anaerobically (Brzezinaska, 1998). Consequently, more soil microbial biomass and microbe exudation resulted in higher soil enzymatic activities in paddy soil (Zeng et al., 2005). Again higher acid phosphatase values were obtained at the initial crop growth period owing to acidic reaction of the soil whereas higher alkaline phosphatase values were obtained towards crop maturity stage (at 90 DAT and harvest) as there was appreciation in soil $\mathrm{pH}$.

\section{References}

Agricultural statistics at glance $2014,1^{\text {st }}$ edition, by Ministry of Agriculture, Oxford University press

Babajideand, P.A., Fagbola, O. 2014. Growth, Yield and Nutrient Uptakes of Sesame (Sesamum indicum) as Influenced by Biofertilizer Inoculants. International Journal of Current Microbiology and Applied Science, 3(8): 859-879.

Brzezinaska, M., Stepniewska, Z., Stepniewski, W. 1998. Soil oxygen status and dehydrogenase activity. Soil Biology and Biochemistry, 30: 1783-1790.

Kizilkaya, R. 2009. Nitrogen fixation capacity of Azotobacter spp. strains isolated from soils in different ecosystems and relationship between them and the microbiological properties of soils. Journal of Environmental Biology, 30(1): 73-82.

Page, A.L., Miller R.H., Keeny, D.R. 1982. Methods of soil and plant analysis, part2, $2^{\text {nd }}$ Edn. No (9) Part in the series, American Society of Agronomy, Inc. Soil Science Society of American Journal. Madison, Wisconsin, U.S.A.

Tabatabai, M.A. 1982. Soil enzymes. Page, A.L., Miller, R.H., Keeney, D.R. (Eds.), Methods of Soil Analysis. Part 2. Chemical and Microbiological Properties. Agronomy No. 9. ASA and SSSA, Madison, WI: 903-948.

Tabatabai, M.A. and Bremner, J.M. 1969. Use of p-nitrophenyl phosphate for assay of soil phosphatase activity. Soil Biology and Biochemistry, 1: 301-307.

Tabatabai, M.A. and Bremner, J.M. 1972. Assay of urease activity in soils. Soil Biology and Biochemistry, 4: 479-487.

Vance, E.D., Brookes, P.C. and Jenkinson, D.S. 1987. An extraction method for measuring soil microbial carbon. Soil Biology and Biochemistry. 19: 703-706.

Zeng, L.S., Liao, M., Chen, C.L. and Huang, C. 2005. Growth Stages of Rice (Oryza sativa) young. Rice Science, 12(4): $283-288$

\section{How to cite this article:}

Soumya Ranjan Dehury, Rajeswari Das, Pinki Seth, Madhusmita Pradhan and Santanu Mohanty. 2018. Effect of Azotobacter vinelandii strain SRIAz3 and N-source on Microbiological Properties of Rice Grown Soil. Int.J.Curr.Microbiol.App.Sci. 7(03): 2170-2178. doi: https://doi.org/10.20546/ijcmas.2018.703.256 